\title{
On two Hamilton cycle problems in random graphs
}

\author{
Alan Frieze* $\quad$ Michael Krivelevich ${ }^{\dagger}$
}

November 16, 2006

\begin{abstract}
We study two problems related to the existence of Hamilton cycles in random graphs. The first question relates to the number of edge disjoint Hamilton cycles that the random graph $G_{n, p}$ contains. $\delta(G) / 2$ is an upper bound and we show that if $p \leq(1+o(1)) \ln n / n$ then this upper bound is tight whp. The second question relates to how many edges can be adversarially removed from $G_{n, p}$ without destroying Hamiltonicity. We show that if $p \geq K \ln n / n$ then there exists a constant $\alpha>0$ such that whp $G-H$ is Hamiltonian for all choices of $H$ as an $n$-vertex graph with maximum degree $\Delta(H) \leq \alpha K \ln n$.
\end{abstract}

\section{Introduction}

In this paper, we give results on two problems related to Hamilton cycles in random graphs.

\subsection{Edge Disjoint Hamilton Cycles}

It was shown by Komlós and Szemerédi [8] that if $p=\frac{\ln n+\ln \ln n+c}{n}$ then,

$$
\lim _{n \rightarrow \infty} \operatorname{Pr}\left(G_{n, p} \text { is Hamiltonian }\right)=\lim _{n \rightarrow \infty} \operatorname{Pr}\left(\delta\left(G_{n, p}\right) \geq 2\right) .
$$

Bollobás [3], Ajtai, Komlós and Szemerédi [1] proved a hitting time version of this statement, i.e., $\mathbf{w h p}^{1}$, as we add random edges $e_{1}, e_{2}, \ldots, e_{m}$ one by one to an empty graph, the graph $G_{m}=$

*Department of Mathematical Sciences, Carnegie Mellon University, Pittsburgh PA 15213, U.S.A. Research supported in part by NSF grant CCR-0200945.

${ }^{\dagger}$ Department of Mathematics, Raymond and Beverly Sackler Faculty of Exact Sciences, Tel Aviv University, Tel Aviv 69978, Israel. E-mail: krivelev@post.tau.ac.il. Research supported in part by USA-Israel BSF Grant $2002-133$ and by grant 526/05 from the Israel Science Foundation.

${ }^{1} \mathrm{~A}$ sequence of events $\mathcal{E}_{n}$ is said to occur with high probability $(\mathbf{w h p})$ if $\lim _{n \rightarrow \infty} \operatorname{Pr}\left(\mathcal{E}_{n}\right)=1$ 
$\left([n],\left\{e_{1}, e_{2}, \ldots, e_{m}\right\}\right)$ becomes Hamiltonian at exactly the point when the minimum degree reaches two.

Let us say that a graph $G$ has property $\mathcal{H}$ if it contains $\lfloor\delta(G) / 2\rfloor$ edge disjoint Hamilton cycles plus a further edge disjoint (near) perfect matching in the case $\delta(G)$ is odd. (Here a (near) perfect matching is one of size $\lfloor n / 2\rfloor)$. Bollobás and Frieze [5] showed that whp $G_{m}$ has property $\mathcal{H}$ as long as the minimum degree is $O(1)$.

It is reasonable to conjecture that $\operatorname{whp} G_{n, p}$ has property $\mathcal{H}$ for any $0 \leq p \leq 1$. Our first result is to show that this is true for $p \leq(1+o(1)) \ln n / n$ which strengthens the non-hitting time version the result quoted from [5].

Theorem 1 Let $p(n) \leq(1+o(1)) \ln n / n$. Then whp $G_{n, p}$ has property $\mathcal{H}$.

We remark that Frieze and Krivelevich [7] showed that if $p$ is constant then whp $G_{n, p}$ almost satisfies $\mathcal{H}$ in the sense that it contains $(1-o(1)) \delta\left(G_{n, p}\right) / 2$ edge disjoint Hamilton cycles.

\subsection{Robustness of Hamiltonicity}

In recent times, there is increasing interest in graphs which are only partially random. For example, Bohman, Frieze and Martin [2] considered graphs of the form $G=H+R$ where $H$ is arbitrary, but with high minimum degree and $R$ is random. In this section we consider graphs of the form $G=R-H$ where $R$ is random and $H$ is an arbitrary subset of $R$, subject to some restrictions. In particular $R=G_{n, p}$

Sudakov and $\mathrm{Vu}[10]$ have recently shown that if $p>(\ln n)^{4} / n$ and if $G=G_{n, p}$ then whp $G-H$ is Hamiltonian for all choices of $H$ as an $n$-vertex graph with maximum degree $\Delta(H) \leq(1 / 2-\varepsilon) n p$. Here $\varepsilon>0$ is an arbitrarily small constant. Note that this bound on $\Delta(H)$ is essentially best possible, otherwise $R-H$ could be a bipartite graph with an uneven partition. In this note we reduce $p$ to $O(\ln n / n)$ but unfortunately, we have to reduce the bound on $\Delta(H)$ as well.

Theorem 2 Let $G=G_{n, p}$ where $p \geq K \ln n / n$ for some sufficiently large constant $K>0$. There exists a constant $\alpha>0$ such that whp $G-H$ is Hamiltonian for all choices of $H$ as an $n$-vertex graph with maximum degree $\Delta(H) \leq \alpha K \ln n$.

\section{Proof of Theorem 1}

\subsection{Preliminaries}

Observe first that the assumption on the edge probability in this theorem can be easily seen to be essentially equivalent to the assumption that the minimum degree $\delta(G)$ of $G_{n, p}$ almost surely satisfies: $\delta(G)=o(\log n)$. 
Notation: For a graph $G=(V, E)$ and two disjoint vertex subsets $U, W$ we denote:

$$
\begin{aligned}
N(U, W) & :=\{w \in W: w \text { has a neighbor in } U\} \\
N(U) & :=N(U, V \backslash U) ; \\
E(U, W) & :=\{e \in E(G):|e \cap U|=1,|e \cap W|=1\} ; \\
e(U, W) & :=|E(U, W)| .
\end{aligned}
$$

Definition 1 A graph $G=(V, E)$ is called a $(k, c)$-expander if $|N(U)| \geq c|U|$ for every subset $U \subseteq V(G)$ of cardinality $|U| \leq k$.

Set

$$
d_{0}=d_{0}(n, p)=\min \left\{k: n\left(\begin{array}{c}
n-1 \\
k
\end{array}\right) p^{k}(1-p)^{n-1-k} \geq 1\right\}
$$

One can prove that whp $\delta\left(G_{n, p}\right)$ satisfies (say):

$$
\left|\delta(G)-d_{0}\right| \leq \ln \ln n
$$

Indeed, $u_{k}=n\left(\begin{array}{c}n-1 \\ k\end{array}\right) p^{k}(1-p)^{n-1-k}$ is the expected number of vertices of degree $k$ and $u_{k+1} / u_{k}=$ $\frac{(n-1-k) p}{(k+1)(1-p)}$. Since $d_{0}=o(\ln n)$ we see that $u_{d_{0}-\ln \ln n}=o(1)$. Furthermore, $u_{d_{0}+\ln \ln n} \rightarrow \infty$ and we can use the Chebyshev inequality to show that $u_{d_{0}+\ln \ln n} \neq 0$ whp.

Define

$$
\rho=\frac{2001\left(d_{0}+\ln \ln n\right)}{n \ln n},
$$

observe that $\rho=o(1 / n)$. Define $p_{0}=p_{0}(n)$ by

$$
1-p=\left(1-p_{0}\right)(1-\rho)
$$

observe that $p_{0}=p-\rho(1-o(1))$. We can thus decompose $G \sim G_{n, p}$ as $G=G_{0} \cup R$, where $G_{0} \sim G_{n, p_{0}}, R \sim G_{n, \rho}$.

Notation. $\delta_{0}=\delta\left(G_{0}\right)$.

Claim 1 For a fixed $G_{0}$, almost surely over the choice of $R \sim G_{n, \rho}, \delta\left(G_{0}\right)=\delta\left(G_{0} \cup R\right)$.

Proof Clearly, $\delta\left(G_{0}\right) \leq \delta\left(G_{0} \cup R\right)$. In the opposite direction, take a vertex $v$ of minimum degree in $G_{0}$. Recall that $\rho=o(1 / n)$, and therefore the edges of $R$ almost surely miss $v$, implying $\delta\left(G_{0} \cup R\right) \leq d_{G_{0} \cup R}(v)=d_{G_{0}}(v)=\delta\left(G_{0}\right)$.

It thus follows that in order to prove Theorem 1 it is enough to prove that almost surely $G_{0} \cup R$ contains $\left\lfloor\delta_{0} / 2\right\rfloor$ disjoint Hamilton cycles, plus an edge disjoint (near) perfect matching if $\delta_{0}$ is odd..

Of course we may (and will indeed) assume that $p(n)=(1+o(1)) \ln n / n$, as otherwise whp $\delta_{0} \leq 1$ and there is nothing new to prove. 


\subsection{Properties of $G_{0}=G_{n, p_{0}}$}

Define

$$
S M A L L=\left\{v \in V: d_{G_{0}}(v) \leq 0.1 \ln n\right\}
$$

Lemma 3 The random graph $G_{0}=G_{n, p_{0}}$, with $p_{0}$ defined by (1), has whp the following properties:

(P1) $G_{0}$ does not contain a path of at most four distinct edges (with possibly identical endpoints), both of whose endpoints lie in SMALL.

(P2) Every vertex has at most one neighbor in $S M A L L$.

(P3) Every set $U \subset V$ of size $|U| \leq 100 n / \ln n$ spans at most $|U|(\ln n)^{1 / 2}$ edges in $G_{0}$.

(P4) For every two disjoint subsets $U, W \subset V$ satisfying: $|U| \leq 100 n / \ln n,|W| \leq 10^{-4}|U| \ln n$,

$$
e_{G_{0}}(U, W)<0.09|U| \ln n .
$$

(P5) For every two disjoint subsets $U, W \subset V$ satisfying: $|U| \geq 100 n / \ln n,|W| \geq n / 4$,

$$
e_{G_{0}}(U, W) \geq 0.1|U| \ln n .
$$

Proof The above are rather standard statements about random graphs, so we will be relatively brief in our arguments.

We start with proving P1. Observe that for a vertex $v \in V\left(G_{0}\right)$, the degree of $v$ is binomially distributed with parameters $n-1$ and $p_{0}$. Therefore,

$$
\begin{aligned}
\operatorname{Pr}[v \in S M A L L] & =\sum_{k \leq 0.1 \ln n} \operatorname{Pr}\left[B\left(n-1, p_{0}\right)=k\right] \leq 0.1 \ln n\left(\begin{array}{c}
n-1 \\
0.1 \ln n
\end{array}\right) p^{0.1 \ln n}(1-p)^{n-1-0.1 \ln n} \\
& \leq 0.1 \ln n\left(\frac{10 e n p}{\ln n}\right)^{0.1 \ln n} e^{-p(n-1-0.1 \ln n)}<29^{0.1 \ln n} e^{-(1-o(1)) \ln n} \\
& <n^{-0.6}
\end{aligned}
$$

Also, for a fixed pair $u \neq v \in V\left(G_{0}\right)$ the probability that $u$ and $v$ are connected by a path of length $\ell$ in $G_{0}$ is at most $n^{\ell-1} p_{0}^{\ell}=((1+o(1)) \ln n)^{\ell} n^{-1}$. Therefore, using the FKG inequality,

$$
\begin{aligned}
\operatorname{Pr}[(u, v \in S M A L L) \&(\operatorname{dist}(u, v) \leq 4)] & \leq \operatorname{Pr}[u \in S M A L L] \operatorname{Pr}[v \in S M A L L] \operatorname{Pr}[\operatorname{dist}(u, v) \leq 4] \\
& \leq 4 \cdot n^{-0.6} \cdot n^{-0.6} \cdot \frac{(1+o(1)) \ln ^{4} n}{n} \\
& <n^{-2.1}
\end{aligned}
$$

Applying the union bound over all possible pairs of distinct vertices $u, v\left(O\left(n^{2}\right)\right.$ of them), we establish $\mathbf{P} 1$. The case where $u=v$ is treated similarly. 
Property $\mathbf{P 2}$ follows directly from P1. Properties P3, P4 are straightforward first moment calculations which we thus omit.

We conclude with proving P5. Fix $U, W$. Then the number of edges between $U$ and $W$ is distributed binomially with parameters $|U||W|$ and $p_{0}$ and has thus expectation $|U||W| p_{0} \geq(1+o(1))|U| \ln n / 4$. Therefore by applying standard Chernoff-type bounds on the lower tail of the binomial distribution, it follows that

$$
\begin{aligned}
\operatorname{Pr}\left[e_{G_{0}}(U, W) \leq 0.1|U| \ln n\right] & \leq \exp \left\{-\frac{(0.25|U| \ln n-0.1|U| \ln n)^{2}}{2 \cdot 0.25|U| \ln n}\right\}=\exp \left\{-2 \cdot 0.15^{2}|U| \ln n\right\} \\
& <\exp \{-4 n\}
\end{aligned}
$$

As the pair $(U, W)$ can be chosen in at most $4^{n}$ ways, $\mathbf{P} 5$ follows by applying the union bound.

\subsection{Pósa's Lemma and its consequences}

Definition 2 Let $G=(V, E)$ be a non-Hamiltonian graph with a longest path of length $\ell$. A pair $(u, v) \notin E(G)$ is called a hole if adding $(u, v)$ to $G$ creates a graph $G^{\prime}$ which is Hamiltonian or contains a path longer than $\ell$. In addition, if the maximum size of a matching in $G$ is $m<\lfloor n / 2\rfloor$ then $(u, v) \notin E(G)$ is called a hole if adding $(u, v)$ to $G$ creates a graph $G^{\prime}$ which is contains a matching of size $m+1$.

Lemma 4 Let $G$ be a non-Hamiltonian connected $(k, 2)$-expander. Then $G$ has a path of length at least $3 k-1$ and at least $k^{2} / 2$ holes.

\section{Proof}

Let $P=\left(v_{0}, \ldots, v_{k}\right)$ be a longest path in graph $G$. A Pósa rotation of $P[9]$ with $v_{0}$ fixed gives another longest path $P^{\prime}=\left(v_{0}, \ldots v_{i} v_{k} \ldots v_{i+1}\right)$ created by adding edge $\left(v_{k}, v_{i}\right)$ and deleting edge $\left(v_{i}, v_{i+1}\right)$. Let $E N D_{G}\left(v_{0}, P\right)$ be the set of endpoints obtained by a sequence of Pósa rotations starting with $P$, keeping $v_{0}$ fixed and using an edge $\left(v_{k}, v_{i}\right)$ of $G$.

Each vertex $v_{j} \in E N D_{G}\left(v_{0}, P\right)$ can then be used as the initial vertex of another set of longest paths whose endpoint set is $E N D_{G}\left(v_{j}, P\right)$, this time using $v_{j}$ as the fixed vertex, but again only adding edges from $G$. Let $E N D_{G}(P)=\left\{v_{0}\right\} \cup E N D_{G}\left(v_{0}, P\right)$.

The Pósa condition (see, e.g., [4], Ch.8.2)

$$
\left|N\left(E N D_{G}(v, P)\right)\right| \leq 2\left|E N D_{G}(v, P)\right|-1
$$

for $v \in E N D_{G}(P)$ together with the fact that $G$ is a $(k, 2)$-expander implies that $\left|E N D_{G}(v, P)\right|>k$. The connectivity of $G$ implies that closing a longest path to a cycle either creates a Hamilton cycle or creates a longer path. For every $v \in E N D_{G}(P)$ and for every $u \in E N D_{G}(v, P)$, a pair $(u, v)$ is a hole. This shows that the number of holes is at least $k^{2} / 2$ (each hole is counted at most twice for both its endpoints). As all neighbors in $G$ of a subset $U \subseteq E N D_{G}(v, P)$ of size $|U|=k$ belong 
to $P$, due to the maximality of $P$, and $G$ is a $(k, 2)$-expander, it follows that the length of $P$ is at least $3 k-1$.

The following lemma is taken from [5].

Lemma 5 Let $G$ be a $(k, 1)$-expander which does not contain a matching of size $\lfloor n / 2\rfloor$. Then $G$ has a matching of size at least $k$ and at least $k^{2} / 2$ holes.

Proof Let $\mathcal{M}$ denote the set of maximum size matchings in $G$ and let $M \in \mathcal{M}$. Fix $v$ uncovered by $M$ and now let $S_{0}$ be the set of vertices reachable from $v$ by an even length alternating path with respect to $M$. Clearly, every vertex of $S_{0}$ is either $v$ or is covered by $M$. Let $x \in N\left(S_{0}\right)$. Then $x$ is covered by $M$, as otherwise we can get a larger matching by using an alternating path from $v$ to $y \in S_{0}$, and then an edge $(y, x)$.

Let $y_{1}$ satisfy $\left(x, y_{1}\right) \in M$. We show that $y_{1} \in S_{0}$. Now there exists $y_{2} \in S_{0}$ such that $\left(x, y_{2}\right) \in E(G)$. Let $P$ be an even length alternating path from $v$ terminating at $y_{2}$. If $P$ contains $\left(x, y_{1}\right)$ we can truncate it to terminate with $\left(x, y_{1}\right)$, otherwise we can extend it using edges $\left(y_{2}, x\right)$ and $\left(x, y_{1}\right)$.

It follows that $\left|N\left(S_{0}\right)\right|<\left|S_{0}\right|$ (as $v \in S_{0}, v$ is not covered by $M$ ). Recalling that $G$ is a $(k, 1$ )expander, we derive that $\left|S_{0}\right|>k$. But then obviously the union $S_{0} \cup N\left(S_{0}\right)$ has at least $2 k$ vertices and thus has at least $2 k-1$ vertices from $M$. This implies: $|M| \geq k$.

Now we prove that $G$ has at least $k^{2} / 2$ holes. Fix $v$ uncovered by $M$ and now let $S \neq \emptyset$ be the other vertices uncovered by $M$. Let $S_{1} \supseteq S$ be the set of vertices reachable from $S$ by an even length alternating path with respect to $M$. As before we can prove that $\left|S_{1}\right|>k$. For every $u \in S_{1}$ there is an even length alternating path with respect to $M$ ending at $u$. Replacing the edges along this path belonging to $M$ with those outside of $M$ gives a maximum matching $M^{\prime} \in \mathcal{M}$ not covering $u$. Thus $(u, v)$ is a hole. Repeating now the above argument with $u, M^{\prime}$ instead of $v, M$, respectively, gives at least $k$ holes touching $u$. Since $\left|S_{1}\right| \geq k$, and each hole is counted at most twice, altogether we get at least $\left|S_{1}\right| k / 2 \geq k^{2} / 2$ holes, as required.

\subsection{Proof idea}

We split the random graph $R$ into $\left\lceil\delta_{0} / 2\right\rceil$ identically distributed random graphs $R_{i}$. We then create $\left\lfloor\delta_{0} / 2\right\rfloor$ Hamilton cycles $H_{i}$ (plus a matching if needed). We use the random edges of $R_{i}$ to fill a hole. Once $H_{i}$ is created its edges are deleted from the graph and we proceed to the next phase. At the $i$-th stage, by the definition of $\delta_{0}$, the graph $G_{i}$ has minimum degree at least 2 , moreover, most vertices in it have degree around $\ln n$ (as each vertex loses at most $\delta_{0}=o(\ln n)$ neighbors during the process), and therefore $G_{i}$ is connected, is an $(n-c n / \ln n, 2)$-expander by properties P1-P5 and has a path $P_{i}$ of length at least $n-c n / \ln n$. We gradually augment $P_{i}$ to a Hamilton path, and then to a Hamilton cycle. At each substage of augmenting $P_{i}$, the current graph has a quadratic number of holes, and therefore a constant number of random edges are expected to augment the current path to a longer one/close a Hamilton cycle. If $\delta_{0}$ is odd, we need a final stage to create a (near) perfect matching. 


\subsection{Formal argument}

We may assume that $\delta_{0} \geq 2$ as otherwise there is nothing new to prove.

Define $\rho_{i}$ by

$$
1-\rho=\left(1-\rho_{i}\right)^{\left\lceil\delta_{0} / 2\right\rceil}
$$

observe that

$$
\rho_{i} \geq \frac{\rho}{\left\lceil\delta_{0} / 2\right\rceil}=\frac{2001\left(d_{0}+\ln \ln n\right)}{\left\lceil\delta_{0} / 2\right\rceil n \ln n} \geq \frac{4000}{n \ln n}
$$

We then represent

$$
R=\bigcup_{i=1}^{\left\lceil\delta_{0} / 2\right\rceil} R_{i}
$$

where $R_{i} \sim G\left(n, \rho_{i}\right)$.

For $i=1, \ldots,\left\lceil\delta_{0} / 2\right\rceil$, let $G_{i}$ be a graph obtained from $G_{0} \cup \bigcup_{j=1}^{i-1} R_{j}$ after having deleted the first $i-1$ Hamilton cycles (assuming that the previous $i-1$ stages were successful, of course). Each vertex $v$ has its degree in $G_{0}$ reduced by at most $2(i-1)$ in $G_{i}$. Therefore if $i \leq\left\lfloor\delta_{0} / 2\right\rfloor$ then the minimum degree $\delta\left(G_{i}\right)$ satisfies $\delta\left(G_{i}\right) \geq \delta_{0}-2(i-1) \geq 2$. If $\delta_{0}$ is odd, then $\delta\left(G_{\left\lceil\delta_{0} / 2\right\rceil}\right) \geq 1$.

We will now show that if $i \leq\left\lfloor\delta_{0} / 2\right\rfloor$ then $G_{i}$ is a $(k, 2)$-expander for $k=n / 3-100 n /(3 \ln n)$. Let $X \subset V$ be a set of $|X|=t$ vertices.

Case 1: $t \leq 100 n / \ln n$.

Denote $X_{0}=X \cap S M A L L,\left|X_{0}\right|=t_{0}, X_{1}=X \backslash X_{0},\left|X_{1}\right|=t_{1}$. Observe first that $\left|N_{G_{i}}\left(X_{0}, V \backslash X\right)\right| \geq$ $2 t_{0}-t_{1}$. Indeed, in $G_{i}$ all edges touching $X_{0}$ have their second endpoint outside $X_{0}$, by Property $\mathbf{P 1}$. We currently have at least two edges per each vertex in $X_{0}$. By Property $\mathbf{P} 2$ each vertex outside $S M A L L$ has at most one neighbor in $X_{0}$ in the graph $G_{i}$. Thus the other endpoints of the edges from $G_{i}$ touching $X_{0}$ are distinct, and at most $t_{1}$ of them land in $X_{1}$.

Now, $X_{1}$ spans at most $t_{1}(\ln n)^{1 / 2}$ edges in $G_{0}$, by Property P3. As the degrees in $G_{0}$ of all vertices in $X_{1}$ are at least $0.1 \ln n$, by the definition of $S M A L L$, at least $0.09 t_{1} \ln n$ edges leave $X_{1}$ in $G_{0}$. But then by Property $\mathbf{P} 4\left|N_{G_{0}}\left(X_{1}\right)\right| \geq 10^{-4} t_{1} \ln n$. By Property $\mathbf{P} 1$ at most $t_{1}$ of those neighbors fall into $X_{0} \cup N_{G_{0}}\left(X_{0}\right)$, implying:

$$
\left|N_{G_{0}}\left(X_{1}, V \backslash X\right)-N_{G_{0}}\left(X_{0}, V \backslash X\right)\right| \geq 10^{-4} t_{1} \ln n-t_{1} .
$$

As in $G_{i}$ every vertex lost at most $\delta_{0}$ neighbors compared to $G_{0}$, we have

$$
\begin{aligned}
\left|N_{G_{i}}\left(X_{1}, V \backslash X\right)-N_{G_{0}}\left(X_{0}, V \backslash X\right)\right| & \geq 10^{-4} t_{1} \ln n-t_{1}-\delta_{0} t_{1} \\
& \geq 10^{-5} t_{1} \ln n .
\end{aligned}
$$

Altogether,

$$
\left|N_{G_{i}}(X)\right| \geq 2 t_{0}-t_{1}+10^{-5} t_{1} \ln n \geq 2 t,
$$

as claimed. 
Case 2: $t \geq 100 n / \ln n$.

Recall that $t \leq n / 3-100 n /(3 \ln n)$. Assume to the contrary that $\left|N_{G_{i}}(X)\right|<2|X|$. Then in $G_{i}$ there is a vertex subset $Y$ disjoint from $X$ such that $|Y|=n-3 t$, and $G_{i}$ has no edges between $X$ and $Y$. But then there were at most $2 \min \left\{\left\lfloor\delta_{0} / 2\right\rfloor|X|,\left\lfloor\delta_{0} / 2\right\rfloor|Y|\right\}$ edges between $X$ and $Y$ in $G_{0}$.

If $t \leq n / 4$, then $n-3 t \geq n / 4$, and we get a contradiction to Property P5 with $X, Y$ substituted for $U, W$, respectively. If $n / 4 \leq t \leq n / 3-(100 n) /(3 \ln n)$, then $n-3 t \geq 100 n / \ln n$, again contradicting Property $\mathbf{P} 5$ with $Y, X$ instead of $U, W$, respectively.

We have proved that given properties P1-P5 of $G_{0}$, for each $i$ the graph $G_{i}$ is deterministically an $(n / 3-100 n /(3 \ln n), 2)$-expander.

A similar argument, in the case where $\delta_{0}$ is odd, shows that the graph $G_{\left\lfloor\delta_{0} / 2\right\rfloor}$ is an $(n / 2-$ $100 n /(2 \ln n), 1)$-expander.

Recall that a random graph $R_{i}$ added at the $i$-th stage is distributed according to $G_{n, \rho_{i}}$ with $\rho_{i} \geq \frac{4000}{n \ln n}$, so $\rho_{i} \geq \frac{120}{n^{2}} \cdot \frac{100 n}{3 \ln n}$ and $\rho_{i}>\frac{20}{n^{2}} \cdot \frac{100 n}{2 \ln n}$. Theorem 1 will thus follow from:

\section{Lemma 6}

(a) Let $G=(V, E)$ be a $(n / 3-k, 2)$-expander on $n$ vertices, where $k=o(n)$. Let $R$ be a random graph $G_{n, p}$ with $p(n)=120 k / n^{2}$. Then

$$
\operatorname{Pr}[G \cup R \text { is not Hamiltonian }]<e^{-\Omega(k)} .
$$

(b) Let $G=(V, E)$ be a $(n / 2-k, 1)$-expander on $n$ vertices, where $k=o(n)$. Let $R$ be a random graph $G_{n, p}$ with $p(n)=20 k / n^{2}$. Then

$$
\operatorname{Pr}[G \cup R \text { does not contain a (near) perfect matching }]<e^{-\Omega(k)} .
$$

\section{Proof}

(a) Observe that by Pósa's Lemma and its consequences (Lemma 4):

- $G$ is connected

(Due to expansion of $G$ there is no room for two connected components);

- $G$ has a path of length at least $n-3 k-1$

(due to Lemma 4);

- If a supergraph of $G_{i}$ is non-Hamiltonian it has at least $(n / 3-k)^{2} / 2>n^{2} / 20$ holes.

We split the random graph $R$ into $6 k$ independent identically distributed graphs

$$
R=\bigcup_{i=1}^{6 k} R_{i}
$$


where $R_{i} \sim G_{n, p_{i}}$ and $p_{i} \geq p /(6 k)=20 / n^{2}$. Set $G_{0}=G$, and for each $i=1, \ldots 6 k$ define

$$
G_{i}=G \cup \bigcup_{j=1}^{i} R_{j}
$$

At Stage $i$ we add to $G_{i-1}$ the next random graph $R_{i}$. A stage $i$ is called successful if a longest path in $G_{i+1}$ is longer than that of $G_{i}$, or if $G_{i+1}$ is already Hamiltonian. Clearly, if at least $3 k+1$ stages are successful then the final graph $G_{6 k}$ is Hamiltonian. Observe that for Stage $i$ to be successful, if $G_{i-1}$ is not yet Hamiltonian, it is enough for the random graph $R_{i}$ to hit one of the holes of $G_{i-1}$. Thus, Stage $i$ is unsuccessful with probability at most $\left(1-p_{i}\right)^{n^{2} / 20}<1 / e$. Let $X$ be the random variable counting the number of successful stages. Then $X$ stochastically dominates $\operatorname{Bin}(6 k, 1-1 / e)$. Hence by standard estimates on the tails of the binomial distribution,

$$
\operatorname{Pr}[G \cup R \text { is not Hamiltonian }] \leq \operatorname{Pr}[X \leq 3 k]<e^{-\Omega(k)},
$$

as claimed.

The proof of (b) is similar.

\section{Proof of Theorem 2}

We will prove the result for $G_{n, m}, m=\frac{1}{2} K n \ln n$. This implies the result for the $G_{n, p}$ model.

This time we will use the coloring argument of Fenner and Frieze [6]. Consider the following properties:

(Q1) $K \ln n / 2 \leq \delta(G) \leq \Delta(G) \leq 2 K \ln n$.

(Q2) $|S| \leq \frac{n}{K^{3}(\ln n)^{2}}$ implies $|E(S)| \leq 2|S|$.

(Q3) $\frac{n}{K^{3}(\ln n)^{2}} \leq|S| \leq n /(K \ln n)$ implies $|N(S)| \geq(K \ln n / 5)|S|$.

(Q4) If $S, T$ are disjoint sets of vertices and $|S| \geq|T| \geq n / 10$ then $e(S, T) \geq(K \ln n / 20)|T|$.

Lemma 7 If $K$ is sufficiently large, $G=G_{n, m}$ satisfies $\mathbf{Q 1 - Q 4} \mathbf{w h p .}$

Proof We will prove that $G_{n, p}$ has these properties where $p=K \ln n / n$. Inflating error probabilities by $O\left(n^{1 / 2}\right)$ will show them for $G_{n, m}$. Q1, Q2 are simple first moment calculations. We will check Q3, Q4. The size of $N(S)$ is distributed as the binomial $B\left(n-s, 1-(1-p)^{s}\right)$. Now $1-(1-p)^{s} \geq s p / 2$ if $s p \leq 1$. Applying a Chernoff bound we see that

$$
\operatorname{Pr}(\exists S \text { failing Q3 }) \leq \sum_{s=\frac{n}{K^{3}(\ln n)^{2}}}^{n /(K \ln n)}\left(\begin{array}{l}
n \\
s
\end{array}\right) e^{-(n-s) s p / 32}=o(1)
$$


Similarly,

$$
\operatorname{Pr}\left(\exists S, T \text { failing Q4) } \leq \sum_{s \geq n / 10} \sum_{t \geq n / 10}\left(\begin{array}{l}
n \\
s
\end{array}\right)\left(\begin{array}{l}
n \\
t
\end{array}\right) e^{-K \ln n|T| / 80}=o(1)\right.
$$

In the following we will asssume that $K$ is sufficiently large and $\alpha$ is sufficiently small so that our claimed inequalities hold. We do not attempt to optimise, since we are far from getting $\alpha$ close to $1 / 2$.

Now let $H$ be a graph with $\Delta(H) \leq \alpha K \ln n$ and let $X$ be any $\beta m$ subset of $E(G-H)$ satisfying $\Delta(X) \leq 2 \beta K \ln n$. Here we will be assuming $1 \gg \beta \gg \alpha$. Let $\Gamma=G-H-X$.

\section{Lemma 8 If Q1-Q4 hold then}

(a) $\Gamma$ is an $(n / 30,2)$-expander.

(b) $\Gamma$ is connected.

\section{Proof}

(a)

(i) $|S| \leq \frac{n}{3 K^{3}(\ln n)^{2}}$.

By construction, we have $\delta(\Gamma) \geq(1 / 2-\alpha-2 \beta) K \ln n$. So if $\left|N_{\Gamma}(S)\right|<2|S|$ we find that $N_{\Gamma}(S) \cup S$ contains at least $((1 / 2-\alpha-2 \beta) K \ln n)|S| / 2$ edges, contradicting Q2.

(ii) $\frac{n}{3 K^{3}(\ln n)^{2}} \leq|S| \leq n /(K \ln n)$.

It follows from Q3 that

$$
\left|N_{\Gamma}(S)\right| \geq((1 / 5-\alpha-2 \beta) K \ln n)|S| \geq 2|S| .
$$

(iii) $n /(K \ln n) \leq|S| \leq n / 30$.

Choose $S^{\prime} \subseteq S$ of size $n /(K \ln n)$. Then

$$
\left|N_{\Gamma}(S)\right| \geq\left|N_{\Gamma}\left(S^{\prime}\right)\right|-|S| \geq(1 / 5-\alpha-2 \beta) n-|S| \geq 2|S| .
$$

(b) It follows from (a) that if $\Gamma$ is not connected then each component is of size at least $n / 10$. But then Q4 implies that there are at least $(1 / 20-\alpha-2 \beta) K|T| \ln n$ edges between each component in $\Gamma$, contradiction.

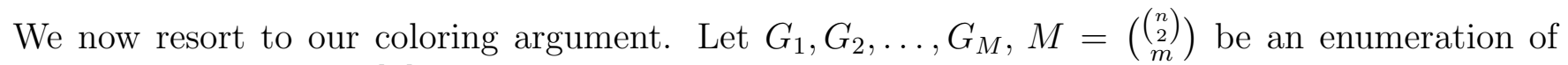
graphs with vertex set $[n]$ and $m$ edges.

For each $i$ let $H_{i}$ be a fixed sub-graph of $G_{i}$ with $\Delta\left(H_{i}\right) \leq \alpha K \ln n$ such that $G_{i}-H_{i}$ is nonHamiltonian, if one exists. Otherwise $H_{i}$ is an arbitrary sub-graph of $G_{i}$ with the same restrictions on the maximum degree. If graph $G$ is non-Hamiltonian, let $\lambda(G)$ denote the length of the longest 
path in $G$ and let $\lambda(G)=n$ if $G$ is Hamiltonian. Now for a graph $G_{i}$, let $X_{i, 1}, X_{i, 2}, \ldots$, be an enumeration of all $\beta m$-subsets of $E\left(G_{i}-H_{i}\right)$. Let $\Gamma_{i, j}=G_{i}-H_{i}-X_{i, j}$. Then let

$$
a_{i, j}= \begin{cases}1 & \left\{\begin{array}{l}
(a) G_{i} \text { satisfies Q1-Q4 } \\
(b) \lambda\left(G_{i}-H_{i}\right)=\lambda\left(G_{i}-H_{i}-X_{i, j}\right) \\
(c) G_{i}-H_{i} \text { is not Hamiltonian } \\
(d) \Delta(X) \leq 2 \beta K \ln n
\end{array}\right. \\
0 \quad \text { otherwise }\end{cases}
$$

The notation $A_{n} \gtrsim B_{n}$ stands for $A_{n} \geq(1-o(1)) B_{n}$.

Lemma 9 If $G_{i}$ satisfies (a) and (c) of (2) then $\sum_{j} a_{i, j} \gtrsim\left(\begin{array}{c}(1-\alpha) m-n \\ \beta m\end{array}\right)$.

Proof $\quad H_{i}$ has at most $\frac{1}{2} \alpha K n \ln n=\alpha m$ edges and to ensure (b) all we have to do is avoid some fixed longest path of $\Gamma_{i, j}$. Furthermore, almost all choices of $\beta m$ edges will induce a sub-graph with maximum degree at most $2 \beta K \ln n$.

Lemma 10 Let $N=\left(\begin{array}{l}n \\ 2\end{array}\right)$. Then,

$$
\sum_{i, j} a_{i, j} \leq m\left(\begin{array}{l}
N \\
m
\end{array}\right)\left(\begin{array}{c}
m \\
\beta m
\end{array}\right)\left(\begin{array}{c}
(1-\beta) m \\
\alpha m
\end{array}\right)(899 / 900)^{\beta m} .
$$

Proof Let $K_{i, j}=G_{i}-X_{i, j}$ and for a fixed graph $K$ with $(1-\beta) m$ edges let us estimate the number of $(i, j)$ with $K_{i, j}=K$ and $a_{i, j}=1$.

For each sub-graph $H \subseteq K$ with $\Delta(H) \leq \alpha K \ln n$, we let $\theta(K, H)$ denote the number of choices of $\beta m$ edges $X$ such that (i) $K+X$ satisfies Q1-Q4 and (ii) $\lambda(K-H)=\lambda(K+X-H)$. Then

$$
\sum_{i, j} a_{i, j} \leq \sum_{K, H} \theta(K, H)
$$

This is because for each $(i, j)$ with $a_{i, j}=1$ there is a corresponding $K_{i, j}=G_{i}-X_{i, j}$ such that $G_{i}=K_{i, j}+X_{i, j}$ satisfies Q1-Q4 and an $H_{i}$ such that $\lambda\left(K_{i, j}-H_{i}\right)=\lambda\left(K_{i, j}+X_{i, j}-H_{i}\right)$.

Now if $K+X$ satisfies Q1-Q4 then from Lemmas 4 and 8 we see that to ensure $\lambda(K-H)=$ $\lambda(K+X-H), X$ must avoid at least $(n / 30)^{2} / 2$ edges i.e.

$$
\theta(K, H) \leq\left(\begin{array}{c}
N-(1-\beta) m \\
\beta m
\end{array}\right)(899 / 900)^{\beta m} .
$$

Consequently,

$$
\begin{aligned}
\sum_{K, H} \theta(K, H) & \leq \sum_{t=0}^{\alpha m}\left(\begin{array}{c}
N \\
(1-\beta) m
\end{array}\right)\left(\begin{array}{c}
(1-\beta) m \\
t
\end{array}\right)\left(\begin{array}{c}
N-(1-\beta) m \\
\beta m
\end{array}\right)(899 / 900)^{\beta m} \\
& \leq m\left(\begin{array}{c}
N \\
(1-\beta) m
\end{array}\right)\left(\begin{array}{c}
(1-\beta) m \\
\alpha m
\end{array}\right)\left(\begin{array}{c}
N-(1-\beta) m \\
\beta m
\end{array}\right)(899 / 900)^{\beta m} \\
& =m\left(\begin{array}{c}
N \\
m
\end{array}\right)\left(\begin{array}{c}
m \\
\beta m
\end{array}\right)\left(\begin{array}{c}
(1-\beta) m \\
\alpha m
\end{array}\right)(899 / 900)^{\beta m} .
\end{aligned}
$$


Let $\nu_{H}$ denote the number of $i$ such that $G_{i}$ satisfies Q1-Q4 and yet $G_{i}-H_{i}$ non-Hamiltonian and let $M=\left(\begin{array}{l}N \\ m\end{array}\right)$. We must show that $\nu_{H}=o(M)$.

It follows from Lemma 9 that

$$
\sum_{i, j} a_{i, j} \gtrsim \nu_{H}\left(\begin{array}{c}
(1-\alpha) m-n \\
\beta m
\end{array}\right) .
$$

On the other hand, Lemma 10 implies

$$
\begin{aligned}
\frac{\nu_{H}}{\left(\begin{array}{c}
N \\
m
\end{array}\right)} & \lesssim \frac{m\left(\begin{array}{c}
m \\
\beta m
\end{array}\right)\left(\begin{array}{c}
(1-\beta) m \\
\alpha m
\end{array}\right)(899 / 900)^{\beta m}}{\left(\begin{array}{c}
(1-\alpha) m-n \\
\beta m
\end{array}\right)} \\
& \leq m\left(\frac{m e}{(1-\alpha) m-n-\beta m}\right)^{\beta m}\left(\frac{(1-\beta) e}{\alpha}\right)^{\alpha m}(899 / 900)^{\beta m} \\
& =o(1),
\end{aligned}
$$

and Theorem 2 follows.

\section{References}

[1] M. Ajtai, J. Komlós and E. Szemerédi, The first occurrence of Hamilton cycles in random graphs, Annals of Discrete Mathematics 27 (1985) 173-178.

[2] T. Bohman, A.M. Frieze and R. Martin, How many random edges make a dense graph Hamiltonian?, Random Structures and Algorithms 22 (2003) 33-42.

[3] B. Bollobás, The evolution of sparse graphs, Graph Theory and Combinatorics. (Proc. Cambridge Combinatorics Conference in Honour of Paul Erdős (B. Bollobás; Ed.)) Academic Press (1984) 35-57.

[4] B. Bollobás, Random graphs, 2nd Ed., Cambridge Univ. Press, Cambridge, 2001.

[5] B. Bollobás and A.M. Frieze, On matchings and hamiltonian cycles in random graphs Annals of Discrete Mathematics 28 (1985) 23-46.

[6] T.I. Fenner and A.M. Frieze, On the existence of hamiltonian cycles in a class of random graphs, Discrete Mathematics 45 (1983) 301-305.

[7] A.M. Frieze and M. Krivelevich, On packing Hamilton Cycles in $\varepsilon$-regular Graphs, Journal of Combinatorial Theory B, 94 (2005) 159-172.

[8] J. Komlós and E. Szemerédi, Limit distributions for the existence of Hamilton circuits in a random graph, Discrete Mathematics 43 (1983) 55-63. 
[9] L. Pósa, Hamiltonian circuits in random graphs, Discrete Mathematics 14 (1976) 359-364.

[10] B. Sudakov and V. Vu, Resilience of graphs, submitted. 